numbers of reflections are $9,1 j$ and $n+1$. This completes the proof for the case of three mirrors.

For more than three mirrors all the considerations preceding equation 2 are valid, but the corresponding inequality has no integer solutions at all.

To prove only Synge's result, which is included above, a much simpler method suffices. If a point arbitrarily near a vertex is reflected successively once in each of the sides meeting there the image remains arbitrarily close to the vertex. The third reflection must bring it to the antipode, an angular distance arbitrarily close to $\pi$ radius. Thus the angular distance from the vertex to the third side is arbitrarily near $\pi / 2$, and this applies to each side. Thus the triangle must be an octant of a sphere, which completes the necessity proof. The sufficiency is proved by reflecting an octant three times in its sides.

It is of interest to compare our results with those described on pps. 81-83 of Coxeter's "Regular Polytopes" (Methuen, London, 1948). There the discrete groups of reflections generated by three or more concurrent planes are deduced. It is first found that all the dihedral angles must be of the form $\pi / n$, then equation 2 is applied, and the solutions are given by equation 3 , with none for more than three planes. In the problem of discrete groups all sequences of reflections are permitted, while in the present problem only "admissible" sequences of reflections are permitted, which is a weaker condition. On the other hand we have the condition that some admissible sequence must carry each point into its antipode, and this condition is strong enough to limit the solutions to those given in equation 4-a subset of the configurations generating discrete groups.

The new parallel reflecting configurations given herein may have practical value in some applications because they reflect back only those rays which are incident from a certain angular region, and the angular region may be made much smaller than that of the mutually orthogonal mirrors.

I wish to express my indebtedness to my colleague, Prof. Wilhelm Magnus, who supplied a crucial part of the necessity proof given above.

\title{
NOTE ON SELF-PROPAGATION OF TURBULENT SPOTS*
}

\section{BY CARL E. PEARSON (Harvard University)}

Introduction. Emmons (1), (2) has recently directed attention towards the manner in which transition occurs from a laminar to a turbulent boundary layer. It appears that the dominating phenomenon is the spontaneous generation of a large number of turbulent "spots" which grow rapidly and eventually coalesce. It has been observed experimentally that, except perhaps immediately after generation, each spot grows in such a manner as to maintain geometric similarity. Because the spot is growing in a boundary laver, the rate at which an elemental portion of its periphery propagates its turbulence outwards is dependent on the orientation of that portion; this dependency of propagation rate on orientation is necessarily associated with the shape of the spot.

It is the purpose of this note to prove the simple but interesting result that for either of two reasonable speculative hypotheses concerning propagation rate, the shape of the spot approaches a certain asymptotic shape which is independent of the initial spot shape.

*Received July 18, 1952. 
Material point hypothesis. It is possible to conceive of the propagation of turbulence into a non-turbulent region as being due to the effert of small random eddies being ('ontinually induced in the quiescent fluid immediately anljoining the turbulent region. In a laminar boundary layer, the propagation of surh induced turbulence may be "onsidered as being at a rate which is dependent on the oricntation of the turbulent surfare in question and in a direction perpendicular to the surface; this leads us to the following equivalent picture.

Suppose that at every instant of time a set of matcrial points occupy a closed convex curve in the plane. Assume that each material point moves perpendicularly to the curve on which it lies and with a velocity $R(\theta)$ depending only on the slope $\theta$ of the curve at that point. Let the family of curves generated in this way be represented parametrically in the complex plane by

$$
z(\theta, t)=x(\theta, t)+i y(\theta, t)
$$

$t$ being the time. Consider first the rate at which the $\theta$ valuc associated with a particular material point changes in time.

In Fig. (1), let $A$ and $B$ be positions orcupicd by the curve at times $t$ and $t+\Delta t$,

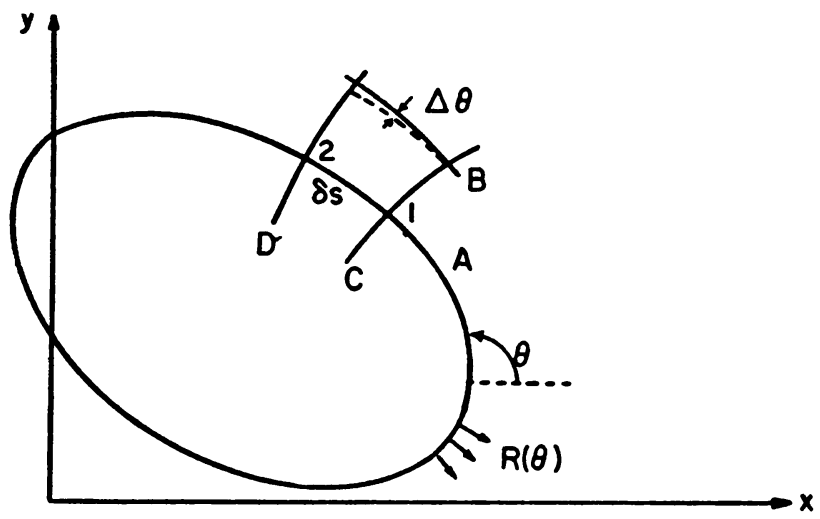

FIG. 1. Neighboring trajectories.

and let $C$ and $D$ be two neighboring orthogonal trajectories (i.e., paths followed by two neighboring particles). Then to the first order, the change in $\theta$ for a particle on trajectory $D$ occurring in a time interval $\Delta t$ is

where

$$
\Delta \theta=-\left(\frac{d R}{d \theta} \delta \theta \Delta t\right) / \delta s,
$$

and therefore

$$
\frac{d R}{d \theta} \delta \theta=R(\theta+\delta \theta)-R(\theta)
$$

$$
\frac{d \theta}{d t}=-\frac{d R}{d \theta} / \frac{d s}{d \theta} .
$$

Now the velocity of the material point is

$$
\frac{d z}{d t}=\frac{\partial z}{\partial t}+\frac{\partial z}{\partial \theta} \frac{d \theta}{d t}
$$


whence

$$
\frac{\partial z}{\partial t}=-i R e^{i \theta}+\left(\frac{d R / d \theta}{\partial x / \partial \theta} \cos \theta\right)\left(\frac{\partial x}{\partial \theta}+i \frac{\partial y}{\partial \theta}\right)=e^{i \theta}\left(\frac{d R}{d \theta}-i R\right)
$$

But the right hand side is a function of $\theta$ alone, and the equation may therefore be integrated to yield

$$
z=e^{i \theta}\left(\frac{d R}{d \theta}-i R\right) t+C(\theta)
$$

where $(:(\theta)$ represents the parametric position of the curve at time $t=0$. Thus the curve approar hes the asymptotic motion

$$
\begin{aligned}
& x=t\left(R \sin \theta+\frac{d R}{d \theta} \cos \theta\right), \\
& y=t\left(\frac{d R}{d \theta} \sin \theta-R \cos \theta\right) .
\end{aligned}
$$

(These two equations may also be used to determine $R$ is the asymptotic shape is known from experiment).

Wavelet hypothesis. Fach point on the periphery of a turbulent spot may be regarded as the continual origin of a disturbance which spreads outwards from it in time; the instantaneous envelope of these disturbances is then the self-propagating curve itself. The shape of the disturbance surrounding any point of origin need not be circular if the region into which it spreads is inhomogeneous (as in the case of a laminar boundary layer). Let the velocity of the (infinitesimal) disturbance be $R(\beta)$ as shown in Fig. (2).

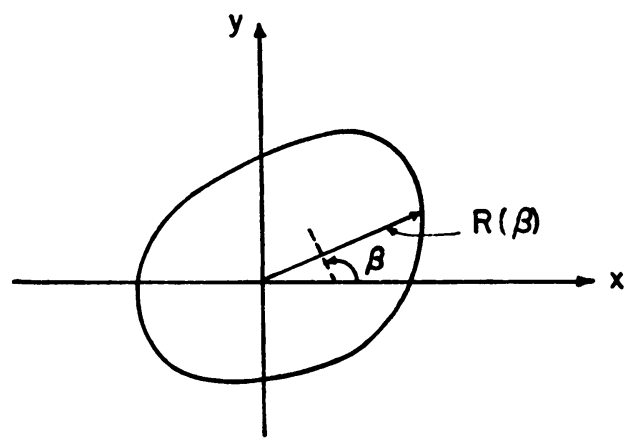

Fig. 2. Wavelet velocity.

Then if the equation of the closed curve is

$$
x=f(\theta, t), \quad y=g(\theta, t),
$$

the equation of a wavelet emanating from the point $(\theta)$ will be, after time $\Delta t$,

$$
\begin{aligned}
& x-f(\theta, t)=\Delta t R(\beta) \sin \beta, \\
& y-g(\theta, t)=\Delta t R(\beta) \cos \beta .
\end{aligned}
$$


The equation of a neighboring wavelet will be

$$
\begin{aligned}
& x-f(\theta+\Delta \theta, t)=\Delta t R(\beta+\Delta \beta) \sin (\beta+\Delta \beta), \\
& y-g(\theta+\Delta \theta, t)=-\Delta t R(\beta+\Delta \beta) \cos (\beta+\Delta \beta),
\end{aligned}
$$

and hence the relation between $\theta$ and $\beta$ is obtained from

$$
\begin{aligned}
& \frac{\partial f}{\partial \theta} \Delta \theta=-\Delta t \frac{\partial}{\partial \beta}(R \sin \beta) \Delta \beta, \\
& \frac{\partial g}{\partial \theta} \Delta \theta=\Delta t \frac{\partial}{\partial \beta}(R \cos \beta) \Delta \beta,
\end{aligned}
$$

whence

$$
\frac{\partial g / \partial \theta}{\partial f / \partial \theta}=\tan \theta=-\frac{\partial(R \cos \beta) / \partial \beta}{\partial(R \sin \beta) / \partial \beta} .
$$

This equation gives the direction $\beta$ of the intersection point of the wavelets as a function of $\theta$.

Now consider again the curve to be composed of material points, this time moving in the $\beta$-direction of Eq. (1).

$$
\begin{aligned}
\frac{d}{d t}(\tan \theta) & =\lim _{\Delta \theta \rightarrow 0, \Delta t \rightarrow 0} \frac{1}{\Delta t}\left\{\frac{y(\theta+\Delta \theta, t+\Delta t)-y(\theta, t+\Delta t)}{x(\theta+\Delta \theta, i+\Delta t)-x(\theta, t+\Delta t)}-\frac{y(\theta+\Delta \theta, t)-y(\theta, t)}{x(\theta+\Delta \theta, t)-x(\theta, t)}\right\} \\
& =\lim _{\Delta t \rightarrow 0} \frac{1}{\Delta t}\left\{\frac{\partial y / \partial \theta-\Delta t(d \beta / d \theta) \partial(R \cos \beta) / \partial \beta}{\partial x / \partial \theta+\Delta t(d \beta / d \theta) \partial(R \sin \beta) / \partial \beta}-\frac{\partial y / \partial \theta}{\partial x / \partial \theta}\right\} \\
& =-\frac{\partial y / \partial \theta}{\partial x / \partial \theta}\left\{\frac{(d \beta / d \theta) \partial(R \cos \beta) / \partial \beta}{\partial y / \partial \theta}+\frac{(d \beta / d \theta) \partial(R \sin \beta) / \partial \beta}{\partial x^{\prime} \partial \theta}\right\} \\
& =-\frac{d \beta / d \theta}{\partial x / \partial \theta}\left\{\frac{\partial}{\partial \beta}(R \cos \beta)+\tan \theta \frac{\partial}{\partial \beta}(R \sin \beta)\right\}=0,
\end{aligned}
$$

by (1). Then

$$
\frac{d x}{d t}=R \sin \beta=\frac{\partial x}{\partial t}+\frac{\partial x}{\partial \beta} \frac{d \beta}{d t}=\frac{\partial x}{\partial t}
$$

whence

$$
x=(R \sin \beta) t+F(\beta) .
$$

and similarly

$$
y=-(R \cos \beta) t+G(\beta),
$$

where $F(\beta)$ and $G(\beta)$ are the parametric representations of the original curve, $\beta$ being now used as the parameter for convenience. Thus there is an asymptotic shape, in fact, that of Fig. (2).

\section{REFERENCES}

(1) H. W. Emmons, The laminar-turbulent transition in a boundary layer, Part I, J. Aero. Sci. 18490 (1951).

(2) H. W. Emmons, Part II, to appear in the Proc. of 1st U. S. National Congress. Theor. Appl. Mech. 